\title{
Epidemiology and risk factors of rectal colonization of carbapenemase-producing Enterobacteriaceae among high-risk patients from ICU and HSCT wards in a university hospital
}

\author{
Li Yan*, Jide Sun, Xiuyu Xu and Shifeng Huang ${ }^{*}$ (i)
}

\begin{abstract}
Background: Nosocomial carbapenemase-producing Enterobacterieceae (CPE) infections constitute a major global health concern and are associated with increased morbidity and mortality. Rectal colonization with CPE is a risk factor for bacterial translocation leading to subsequent endogenous CPE infections. This prospective observational study was aimed to investigate the prevalence and epidemiology of rectal colonization of CPE, the carbapenemase genotypes, and to identify the independent risk factors for the acquisition of CPE colonization in high-risk patients from ICU and HSCT wards in a university hospital in China.

Methods: In a prospective cohort study, 150 fecal samples from rectal swabs were consecutively obtained for inpatients from the intensive care unit (ICU) and hematopoietic stem cell transplantation (HSCT) wards from November 2018 to May 2019, and screening test for CPE was conducted by using prepared in-house trypsin soybean broth (TSB) selective media and MacConkey agar. Antimicrobial susceptibility was determined by the broth microdilution method and carbapenemase genes were characterized by both the GeneXpert Carba-R and PCR for bla $a_{\mathrm{KPC}}, b / a_{\mathrm{NDM}}$, bla $a_{\mathrm{IMP}}$, bla $a_{\mathrm{VIM}}$ and blaOXA. Multi-locus sequence typing (MLST) was employed to characterize the genetic relationships among the carbapenemase-producing K. Pneumonia (CPKP) isolates. In order to further investigate the risk factors and clinical outcomes of CPE colonization, a prospective case-control study was also performed.

Results: Twenty-six suspected CPE strains, including 17 Klebsiella pneumoniae, 6 Escherichia coli, 1 Citrobacter freundii, 1 Enterobacter Kobe, and 1 Raoultella ornithinolytica, were identified in 25 non-duplicated rectal swab samples from 25 patients, with a carriage rate of $16.67 \%$ (25/150). Through GeneXpert Carba-R and subsequent PCR and sequencing, all the suspected CPE isolates were identified to be positive for the carbapenemase genes, of which 17 were bla KPC $^{-}$

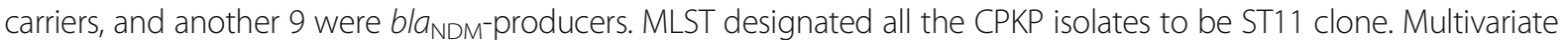
analysis indicated that urinary system diseases, operation of bronchoscopy, and combined use of antibiotics were independent risk factors for acquiring CPE colonization in high-risk patients from the ICU and HSCT wards.

(Continued on next page)
\end{abstract}

\footnotetext{
*Correspondence: cqyanli2000@126.com; sfhuang@hospital.cqmu.edu.cn Department of Laboratory Medicine, the First Affiliated Hospital of Chongqing Medical University, No.1 Friendship Road, Yuzhong District, Chongqing 400016, China
}

(c) The Author(s). 2020 Open Access This article is licensed under a Creative Commons Attribution 4.0 International License, which permits use, sharing, adaptation, distribution and reproduction in any medium or format, as long as you give appropriate credit to the original author(s) and the source, provide a link to the Creative Commons licence, and indicate if changes were made. The images or other third party material in this article are included in the article's Creative Commons licence, unless indicated otherwise in a credit line to the material. If material is not included in the article's Creative Commons licence and your intended use is not permitted by statutory regulation or exceeds the permitted use, you will need to obtain permission directly from the copyright holder. To view a copy of this licence, visit http://creativecommons.org/licenses/by/4.0/. The Creative Commons Public Domain Dedication waiver (http://creativecommons.org/publicdomain/zero/1.0/) applies to the data made available in this article, unless otherwise stated in a credit line to the data. 
(Continued from previous page)

Conclusions: This study revealed a high prevalence of rectal CPE colonization in high-risk patients from ICU and HSCT wards, and a predominant colonization of the KPC-producing K. pneumoniae clone ST11. Stricter infection control measures are urgently needed to limit the dissemination of CPE strains, especially in patients who were afflicted by urinary system diseases, have underwent bronchoscopy, and were previously exposed to combined antibiotic use.

Keywords: Fecal carriage, CRE, CPE, KPC, Risk factors

\section{Background}

Carbapenem-resistant Enterobacteriaceae (CRE) is a serious public health threat worldwide [1,2]. CRE infections have been emerging rapidly and posing serious challenges to the clinical management [3], thus, timely and efficient diagnosis, strict and evidence-based infection control measures, and prompt and effective therapy are of paramount importance. To help direct research and development efforts toward the production of novel drugs, CRE was recently listed as one of the three critical-priority pathogens by the World Health Organization (WHO) $[4,5]$.

Resistance to carbapenems in Enterobacteriaceae occurs via one or a combination of carbapenemase production, production of ESBLs and/or AmpC in combination with porin loss/deficiency, carbapenem efflux, or mutations in penicillin-binding proteins (PBPs) [6, 7], among which carbapenemase gene acquisition is of greatest concern [8], as it most frequently occurs via transfer of plasmids, which usually co-harbor $\beta$-lactamases and other resistance determinants, rendering these carbapenemase-producing Enterobacteriaceae (CPE) strains multi-drug resistant (MDR) or extensively drug-resistant (XDR) [9].

$\mathrm{CPE}$ is the most problematic due to higher-level antimicrobial resistance, clonal spread and horizontal gene transfer [10]. Carbapenemases include Ambler class A $\beta$ lactamases (e.g., KPC and GES), class B metallo- $\beta$ lactamases (MBLs, e.g., NDM, VIM, IMP, and SPM), and class D $\beta$-lactamases (e.g., OXA-48 and OXA-181) [6]. In the case of CPE, only some "second-line" drugs, such as polymyxins, tigecycline, and fosfomycin, and some "last resort" antibiotics, such as aztreonam/avibactam [11], ceftazidime/avibactam [12], meropenem/vaborbactam [13], and imipenem/relebactam [14] have been demonstrated to be effective in some cases. In addition, although several newer investigational agents that have not trialed against CPE yet, such as eravacycline [15], plazomicin [16], and cefiderocol [17], may be effective against $\mathrm{CPE}$, but is still speculative. Also, resistance to these new drugs can be expected to emerge, as has been seen with aztreonam/avibactam [18] and ceftazidimeavibactam [19]. It is therefore crucial to implement efficient infection control measures to limit the spread of these pathogens.

The lack of control measures directed at CPE carriers has led to the spread of CPE in healthcare settings.
Screening for asymptomatic CPE colonization and implementing contact precautions will reduce patientto-patient transmission. Although evidence-based guidelines on prevention and control of CPE are available [20-22], CPE still remains as a severe challenge in health care settings, and more studies on appropriate countermeasures are required especially for high-risk patients from intensive care unit (ICU) and hematopoietic stem cell transplantation (HSCT) wards. This prospective observational study was aimed to investigate the prevalence and epidemiology of fecal carriage of CPE, the carbapenemase genotypes, and to identify the independent risk factors for the acquisition of CPE colonization in highrisk patients in ICU and HSCT wards from a university hospital in China.

\section{Methods}

\section{Setting and study design}

The present study was a single-center prospective survey performed at the first Affiliated Hospital of Chongqing Medical University, China. Our hospital is a 3200-bed teaching hospital providing all types of acute care with around 200,000 admissions and 1000,000 hospital-days per year.

We implemented a prospective analysis of a cohort of CPE-colonized patients detected from November 2018 to May 2019. All hospitalized patients in ICU wards (including central ICU, respiratory ICU, critical surgery ICU, neurology ICU, neurosurgery ICU, thoracic and cardiac surgery ICU) and HSCT wards during this period were included, due to the high prevalence of CRE carriage observed in these wards in the baseline survey, and are thus considered the highest-risk sites for CRE transmission.

Rectal swabs were obtained from all patients in a representative sample of wards in ICU and HSCT. Each cohort patient was systematically screened for CPE colonization on the day of admission. A total of 150 rectal swabs were collected from non-repetitive patients. Stool samples collected from rectal swab were cultured on selective TSB media (Pangtong, China), and the suspected $\mathrm{CPE}$ isolates were further identified at the species level by the VITEK MS (bioMérieux, MO, USA) system, and routine antimicrobial susceptibility testing was 
performed using the VITEK2 compact (bioMérieux, Inc., NC, USA) system.

\section{Bacterial identification and antimicrobial susceptibility testing}

A total of 26 clinical CRE strains were isolated and identified from November 2018 to May 2019 by using the VITEK MS (bioMerieux, Hazelwood, MO, United States) automated system at the department of laboratory medicine, all of which were resistant to at least one carbapenem on the basis of antimicrobial susceptibility testing results determined by the broth microdilution method, with the criteria of $\mathrm{MIC}$ of $\geq 2 \mathrm{mg} / \mathrm{L}$ for ertapenem, $\geq 4$ $\mathrm{mg} / \mathrm{L}$ for imipenem, and $\geq 4 \mathrm{mg} / \mathrm{L}$ for meropenem.

\section{Detection of antibiotic resistance genes}

Both GeneXpert Carba-R (bla $a_{\mathrm{KPC}}, b l a_{\mathrm{NDM}}, b l a_{\mathrm{IMP}}, b l a-$ VIM and $\left.b l a_{\text {OXA }}\right)$ and PCR were used to confirm the suspected resistance phenotypes. Moreover, the Carba NP test and SCIM were performed on all isolates to determine whether any bacteria produced carbapenemases by phenotypic methods but were negative by genotypic methods, or vice versa.

Multi-locus Sequence Typing (MLST) of the 17 bla $a_{\mathrm{KPC}}$-carrying $K$. pneumoniae isolates.

In order to further characterize the genetic relationships among the $17 b l a_{\mathrm{KPC}}$-carrying carbapenemaseproducing K. Pneumonia (CPKP) isolates, multi-locus sequence typing (MLST) was performed by the amplification and subsequent sequencing of the internal fragments of seven housekeeping genes of K. Pneumonia isolates (rpoB, gapA, mdh, pgi, phoE, infB, and tonB) and comparing them with KP MLST database (https:// bigsdb.pasteur.fr/klebsiella/klebsiella.html) to determine the allele types and STs of CPKP isolates.

\section{Risk factors for CPE colonization}

We conducted a prospective case-control study to explore the risk factors and clinical outcomes of patients colonized with CPE from November 2018 to June 2019 in Chongqing, China. Patients with CPE colonization were included as cases. Controls were identified as patients without CPE colonization with well-balanced demographic characteristics, pre-existing medical conditions, and immune-compromising comorbidities as compared with the cases. Clinical and epidemiological data, including the demographics, underlying diseases, the primary diagnosis at admission, invasive procedures prior to the isolation of $\mathrm{CPE}$, previous exposures of antibiotic within 3 months, and the clinical outcomes, were extracted from the patients' electronic medical records system and clinical microbiology laboratory database.

\section{Statistical analysis}

Data were collected using Excel software (Microsoft, Redmond, CA, USA), and were described using the mean with standard deviation (SD) for continuous variables and proportions (\%) for qualitative variables.

All analyses were performed using SPSS v.22.0 software (SPSS Inc., Chicago, IL, United States). Univariate analyses were performed separately for each of the variables. Categorical variables were compared using a chisquare test or Fisher's exact test as appropriate. Continuous variables were compared using Student's t-test (normally distributed variables) and Wilcoxon rank-sum test (non-normally distributed variables) as appropriate. The odds ratio (OR) and 95\% confidence interval (CI) were calculated to evaluate the strength of any association. Variables with $P \leq 0.05$ on univariate analysis were evaluated as potential covariates in a stepwise multivariate logistic regression model. All $P$-values were two sided, and $P<0.05$ was considered significant.

\section{Ethical considerations}

The data and samples analyzed in the present study were obtained in accordance with the standards and approved by the Chongqing Medical University Institutional Review Board and Biomedical Ethics Committee.

\section{Results}

Prevalence of positive CRE colonization among high-risk patients from ICU and HSCT wards

In the CRE prevalence survey, 150 patients from ICU and HSCT wards were prospectively recruited, and a total of 150 non-repetitive fecal swabs were collected, among which 26 CRE strains from 25 individual patients were detected as being CRE positive, with a carriage rate of $16.67 \%$ (25/150). Among the 26 CRE isolates, $22 \mathrm{CRE}$ isolates $(84.62 \%, 22 / 26)$ were isolated from 21 individual patients in the ICU wards, with a carriage rate of $18.58 \%$ (21/113); another 4 were from 4 patients in the HSCT wards, with a carriage rate of $10.81 \%$ (4/37). The 26 isolated CRE strains included 17 Klebsiella pneumoniae (65.38\%), 6 Escherichia coli (23.08\%), 1 Citrobacter freundii (3.85\%), 1 Enterobacter Kobe (3.85\%), and 1 Raoultella ornithinolytica (3.85\%). The 26 CRE strains were isolated from 25 non-duplicated samples from 25 individual patients (14 male and 11 female; 21 from ICU and 4 from HSCT) whose mean age was $63.48 \pm 22.81$ years. Among these patients, $40.0 \%(10 / 25)$ were transferred from another hospital.

\section{Confirmation of carbapenemase-producing phenotypes and molecular characterization of carbapenemase genes} Both Carba NP test and sCIM were performed on all isolates to determine whether the CRE isolates produced carbapenemases by phenotypic methods. Among the 26 
CRE isolates, all were positive in both the two carbapenemase phenotype confirmatory tests, in concordance with our later molecular characterization results from both GeneXpert Carba-R and subsequent PCR and sequencing, which demonstrated $26 \mathrm{CPE}$ isolates with carbapenemase genes. As was shown in Table 1, the most prevalent carbapenemase gene was bla $a_{\mathrm{KPC}}(65.38 \%, 17 /$ 26), detected in all the $17 \mathrm{~K}$. pneumoniae isolates; followed by bla $a_{\mathrm{NDM}}(34.62 \%, 9 / 26)$, detected in all of the 6 Escherichia coli isolates, 1 Citrobacter freundii, 1 Enterobacter Kobe, and 1 Raoultella ornithinolytica isolate; bla $_{\mathrm{IMP}}, \quad b l a_{\mathrm{VIM}}$, and $b l a_{\mathrm{OXA}-48}$ were not detected (Table 1). Notably, all the $17 \mathrm{~K}$. pneumoniae isolates harbored $b l a_{\mathrm{KPC}}$, while all the 6 carbapenemase-producing Escherichia coli strains carried bla $a_{\mathrm{NDM}}$. It's also worth noting that one patient was demonstrated to co-harbor two CPE colonization isolates, with one $K$. pneumoniae strain producing $\mathrm{KPC}$, and another E. coli isolate expressing NDM. Moreover, all the 26 CPE isolates showed resistance to all the three tested carbapenems (imipenem, meropenem, and ertapenem), showing a perfect correlation between CPE phenotype and genotype.

\section{MLST typing results}

To further characterize the genetic relationships among the 17 bla $_{\mathrm{KPC}}$-carrying CPKP isolates, MLST was carried out. All the $17 b l a_{\mathrm{KPC}^{-}}$-carrying CPKP isolates were exclusively assigned to ST11, clearly demonstrating that resistance is restricted to one genetic background and that it is a problem of inter-hospital spread of one clone.

\section{Risk factors for the acquisition of rectal CPE colonization in high-risk patients from ICU and HSCT wards}

The risk factors for the acquisition of rectal CPE colonization in high-risk patients from ICU and HSCT wards were shown in Table 2. The univariate analysis indicated that higher APACHE II Scores, diabetes mellitus, urinary system disease, coronary heart disease, endocrine system diseases, multiple organ dysfunction syndrome, bronchoscopy, and combined use of antibiotics were significantly more frequent in patients with rectal CPE colonization $(P<=0.05)$. Further multivariate logistic regression analysis demonstrated that urinary system

Table 1 Molecular characterization of carbapenemase genes in 26 CRE isolates as detected by GeneXpert Carba-R and PCR

\begin{tabular}{|c|c|c|c|c|c|}
\hline \multirow[t]{2}{*}{ Bacteria } & \multicolumn{5}{|c|}{ Carbapenemases (isolate number) } \\
\hline & $b / a_{\mathrm{KPC}}$ & $b l a_{\mathrm{NDM}}$ & $b / a_{\mathrm{VIM}}$ & $b l a_{I M P}$ & $b / a_{\mathrm{OXA}-48}$ \\
\hline Klebsiella pneumoniae & 17 & - & - & - & - \\
\hline Escherichia coli & - & 6 & - & - & - \\
\hline Citrobacter freundii & - & 1 & - & - & - \\
\hline Enterobacter Kobe & - & 1 & - & - & - \\
\hline Raoultella ornithinolytica & - & 1 & _- & - & _- \\
\hline
\end{tabular}

disease (OR [Odd ratio]: 18.06, 95\% CI [Confidence Interval]: $3.31-98.62, p=0.001)$, operation of bronchoscopy (OR: $4.05,95 \%$ CI: $1.30-12.60, p=0.01$ ), and previous exposure to combined antibiotics (OR: $3.60,95 \% \mathrm{CI}$ : 1.18-10.93, $p=0.02$ ) were independent risk factors for the acquisition of rectal $\mathrm{CPE}$ colonization in high-risk patients from ICU and HSCT wards.

\section{Discussion}

CPE transmission has been enabled by the lack of control measures directed at CPE carriers in healthcare settings. Screening patients for asymptomatic colonization and implementation of contact precautions could reduce patient-to-patient transmission [23]. Investigation of the prevalence of the rectal CPE colonization among highrisk patients from ICU and HSCT wards can contribute to better infection control measures to limit CPE dissemination. Our study revealed that the rate of rectal CPE colonization in the high-risk patients from ICU and HSCT wards was $16.67 \%$, with the sole carbapenemase gene being bla $a_{\mathrm{KPC}}$ in $K$. pneumoniae isolates and $b l a_{\mathrm{NDM}}$ in E. coli. Stricter infection control measures are urgently needed to limit the CPE dissemination, especially in patients who were afflicted by the urinary system diseases, have underwent bronchoscopy, and were previously exposed to combined antibiotic use.

While CRE infection rates were reported to be varied (from 7.6 to $44.4 \%$ ) in individual studies for CREcolonized patients $[24,25]$, a recent meta-analysis suggested an overall $16.5 \%$ risk of infection with CRE amongst patients colonized with CRE [26]. From a clinical point of view, all patients in whom colonization could represent a risk factor for invasive disease should be screened [27]. Notably, chemotherapy for acute leukemia, solid organ transplantation, and ICU stay were identified as the most important patient-related conditions associated with a significant risk of CRE infections in colonized patients [28]. Accordingly, with this indication, ICUs, Transplant Units, Hematological Units, major surgical and Infectious Disease Units represent the preferential setting for active targeting screening. We thus focused on the investigation of the prevalence of CPE colonization in patients from ICU and HSCT wards in our hospital. As expected, this study revealed a higher rate of fecal carriage of CPE (16.67\%) in the highrisk patients from ICU and HSCT wards than that was reported for randomly recruited patients from several previous studies in Fujian (6.6\%) [29], Hunan (8.5\%) [30], and Thailand (8.7\%) [31].

Rapid detection and characterization of carbapenemase types in colonized CPE strains according to the Ambler classification will lead to improved guidance on the implementation of infection control measures. In this study, we have demonstrated that carbapenem 
Table 2 Univariate and multivariate analyses of risk factors for patients with CPE colonization

\begin{tabular}{|c|c|c|c|c|c|c|}
\hline \multirow[t]{2}{*}{ Variable } & \multirow{2}{*}{$\begin{array}{l}\text { CRE } \\
\text { Colonization } \\
(n=25)\end{array}$} & \multirow{2}{*}{$\begin{array}{l}\text { Control } \\
(n=75)\end{array}$} & \multicolumn{2}{|c|}{ Univariate analysis } & \multicolumn{2}{|c|}{ Multivariate analysis } \\
\hline & & & OR $(95 \% \mathrm{CI})$ & $P$-value & OR $(95 \% \mathrm{Cl})$ & $P$-value \\
\hline \multicolumn{7}{|l|}{ Patient characteristics } \\
\hline Man gender (Male) & $14(56.00 \%)$ & $48(64.00 \%)$ & $0.65(0.21-2.00)$ & 0.45 & & \\
\hline Age & $63.48 \pm 22.81$ & $60.76 \pm 19.01$ & $1.01(0.98-1.05)$ & 0.36 & & \\
\hline Transferring from another hospital & $10(40.00 \%)$ & $37(49.33 \%)$ & $0.92(0.29-2.92)$ & 0.88 & & \\
\hline \multicolumn{7}{|l|}{ Underlying diseases } \\
\hline PITT Scores & $2.28 \pm 1.86$ & $2.98 \pm 2.09$ & $0.79(0.54-1.15)$ & 0.22 & & \\
\hline APACHE II Scores & $17.16 \pm 7.18$ & $15.74 \pm 8.13$ & $1.10(1.01-1.20)$ & 0.05 & $0.40(0.09-1.71)$ & 0.22 \\
\hline Hypertension & $13(52.00 \%)$ & $38(50.66 \%)$ & $0.62(0.18-2.15)$ & 0.42 & & \\
\hline Diabetes & $12(48.00 \%)$ & $20(26.67 \%)$ & $3.97(1.16-13.62)$ & 0.03 & $4.30(0.67-27.306)$ & 0.12 \\
\hline Tuberculosis & $2(8.00 \%)$ & $4(5.33 \%)$ & $0.94(0.10-8.50)$ & 0.95 & & \\
\hline Hepatitis B & $2(8.00 \%)$ & $3(4.00 \%)$ & $2.17(0.24-19.57)$ & 0.09 & & \\
\hline Tumour & $12(48.00 \%)$ & $21(28.00 \%)$ & $2.88(0.85-9.72)$ & 0.09 & & \\
\hline Respiratory diseases & $17(68.00 \%)$ & $36(48.00 \%)$ & $2.64(0.76-9.14)$ & 0.13 & & \\
\hline COPD & $9(36.00 \%)$ & $5(6.67 \%)$ & $0.92(0.20-4.20)$ & 0.91 & & \\
\hline Hepatobiliary disease & $8(32.00 \%)$ & $26(34.67 \%)$ & $0.63(0.18-2.10)$ & 0.47 & & \\
\hline Gastrointestinal disease & $6(24.00 \%)$ & 27 (36.00\%) & $0.36(0.09-1.46)$ & 0.15 & & \\
\hline Chronic kidney disease & $6(24.00 \%)$ & $21(28.00 \%)$ & $0.75(0.21-2.70)$ & 0.66 & & \\
\hline Urinary system disease & $10(40.00 \%)$ & $6(8.00 \%)$ & $7.71(2.21-26.83)$ & 0.001 & $18.06(3.31-98.62)$ & 0.001 \\
\hline Cardiovascular disease & $15(60.00 \%)$ & $41(54.67 \%)$ & $0.87(0.27-2.80)$ & 0.82 & & \\
\hline Coronary heart disease & $7(28.00 \%)$ & $10(13.33 \%)$ & $4.26(1.12-16.28)$ & 0.03 & $3.29(0.91-11.98)$ & 0.07 \\
\hline Neurological disease & $9(36.00 \%)$ & $18(24.00 \%)$ & $2.27(0.64-7.99)$ & 0.20 & & \\
\hline Disease of immune system & $1(4.00 \%)$ & $5(6.67 \%)$ & $0.22(0.02-2.85)$ & 0.25 & & \\
\hline Hematological system diseases & $4(16.00 \%)$ & $16(21.33 \%)$ & $1.47(0.32-6.72)$ & 0.62 & & \\
\hline Endocrine System Diseases & $15(60.00 \%)$ & $28(37.33 \%)$ & $3.16(1.05-9.54)$ & 0.04 & $1.62(0.33-7.99)$ & 0.55 \\
\hline Surgery in the past 6 months & $2(8.00 \%)$ & $16(21.33 \%)$ & $0.32(0.05-1.99)$ & 0.22 & & \\
\hline Multiple organ dysfunction syndrome & $6(24.00 \%)$ & $8(10.67 \%)$ & $6.27(1.35-29.05)$ & 0.02 & & \\
\hline Transplant & $4(16.00 \%)$ & $11(14.67 \%)$ & $1.82(0.43-7.64)$ & 0.42 & & \\
\hline Urinary tract infection & $10(40.00 \%)$ & $12(16.00 \%)$ & $3.64(0.78-16.87)$ & 0.09 & & \\
\hline Respiratory infection & $21(84.00 \%)$ & $51(68.00 \%)$ & $2.20(0.37-12.92)$ & 0.38 & & \\
\hline Abdominal infection & $8(32.00 \%)$ & $2(2.67 \%)$ & $0.48(0.05-4.68)$ & 0.53 & & \\
\hline Septic shock & $1(4.00 \%)$ & $9(12.00 \%)$ & $0.99(0.95-1.02)$ & 0.53 & & \\
\hline Severe anemia & $5(20.00 \%)$ & $17(26.15 \%)$ & $1.06(0.27-4.13)$ & 0.94 & & \\
\hline Glucocorticoid & $7(28.00 \%)$ & $12(16.00 \%)$ & $2.58(0.86-9.72)$ & 0.21 & & \\
\hline Cardiotonic & $2(8.00 \%)$ & $5(6.67 \%)$ & $0.78(0.08-7.87)$ & 0.84 & & \\
\hline Receipt of total parenteral Nutrition (days) & $39.00 \pm 57.47$ & $16.71 \pm 25.33$ & $0.99(0.95-1.02)$ & 0.53 & & \\
\hline Urinary catheter (days) & $56.08 \pm 60.35$ & $21.80 \pm 26.07$ & $1.04(0.99-1.09)$ & 0.08 & & \\
\hline Nasal catheter (days) & $35.38 \pm 48.75$ & $21.59 \pm 36.98$ & $0.97(0.92-1.04)$ & 0.16 & & \\
\hline Mechanical ventilation (days) & $34.64 \pm 48.61$ & $15.15 \pm 19.01$ & $1.02(0.98-1.09)$ & 0.16 & & \\
\hline Trachea cannula & $24.80 \pm 48.02$ & $12.65 \pm 24.27$ & $0.98(0.95-1.03)$ & 0.55 & & \\
\hline Tracheotomy & $10(40.00 \%)$ & $31(41.33 \%)$ & $0.17(0.02-1.28)$ & 0.08 & & \\
\hline Bronchoscopy & $12(48.00 \%)$ & $13(17.33 \%)$ & $8.15(1.61-41.22)$ & 0.01 & $4.05(1.30-12.60)$ & 0.01 \\
\hline Drainage tube & $19(76.00 \%)$ & $55(73.33 \%)$ & $0.31(0.05-1.76)$ & 0.18 & & \\
\hline Penicillin & $5(20.00 \%)$ & $17(22.67 \%)$ & $0.60(0.14-2.55)$ & 0.48 & & \\
\hline
\end{tabular}


Table 2 Univariate and multivariate analyses of risk factors for patients with CPE colonization (Continued)

\begin{tabular}{|c|c|c|c|c|c|c|}
\hline \multirow[t]{2}{*}{ Variable } & \multirow{2}{*}{$\begin{array}{l}\text { CRE } \\
\text { Colonization } \\
(n=25)\end{array}$} & \multirow{2}{*}{$\begin{array}{l}\text { Control } \\
(n=75)\end{array}$} & \multicolumn{2}{|c|}{ Univariate analysis } & \multicolumn{2}{|c|}{ Multivariate analysis } \\
\hline & & & OR $(95 \% \mathrm{Cl})$ & $P$-value & OR $(95 \% \mathrm{Cl})$ & $P$-value \\
\hline Cephalosporins & $8(32.00 \%)$ & $25(33.33 \%)$ & $0.37(0.08-1.61)$ & 0.19 & & \\
\hline Carbapenem & $10(40.00 \%)$ & $24(32.00 \%)$ & $1.12(0.32-3.84)$ & 0.86 & & \\
\hline Fluoroquinolone & $5(20.00 \%)$ & $11(14.67 \%)$ & $0.87(018-4.30)$ & 0.87 & & \\
\hline Tetracycline & $3(12.00 \%)$ & $4(5.33 \%)$ & $0.93(0.14-5.90)$ & 0.92 & & \\
\hline Glycopeptide & $3(12.00 \%)$ & $12(16.00 \%)$ & $0.20(0.03-1.15)$ & 0.07 & & \\
\hline Tigecycline & $4(16.00 \%)$ & $3(4.00 \%)$ & $1.94(0.27-13.98)$ & 0.51 & & \\
\hline Combined use of antibiotics & $18(72.00 \%)$ & $32(42.67 \%)$ & $5.91(1.20-29.16)$ & 0.03 & $3.60(1.18-10.93)$ & 0.02 \\
\hline
\end{tabular}

resistance in rectal $K$. pneumoniae and E. coli isolates was respectively solely associated with KPC and NDM production. On the other hand, as selective pressure from carbapenem use continues, multiple carbapenemases per pathogen, including pathogens carrying three different carbapenemase genes [32, 33], or multiple CPE pathogens per patient are increasingly common. In our screening, although non CPE isolates co-harboring double or triple carbapenemases were identified, we witnessed one patient co-colonized by two CPE isolates, with one KPC-producing $K$. pneumoniae strain and another NDM-expressing E. coli strain. As these deleterious pathogens may spread throughout health care facilities, closer attention to infection control measures and stewardship of the carbapenem-containing drugs should be paid in order to control selection of even more detrimental pathogens.

CPKP strains are the most clinically prominent CPE. Ever since the first report of CPKP in 2001 from the United States [34], an increase in CPKP isolates, largely attributed to ST11, ST15, ST101, ST258, and ST512 strains, was reported across Europe [35]. CPKP isolates emerging from the Indian Ocean rim [36], the United States [37], and China [38] have further exacerbated the global burden of CPKP. The genetic relationship among the $17 \mathrm{KPC}$-producing CPKP clones was tracked by MLST. Surprisingly, all the $17 b l a_{\mathrm{KPC}}$-carrying CPKP isolates were exclusively designated as Clone ST11, indicating that carbapenem-resistance in $K$. pneumoniae is limited to clone ST11. Although the dissemination mechanism is still unclear, our findings highlighted the importance of long-term active resistance surveillance of CPKP in high-risk patients from both ICU and HSCT wards.

High prevalence of CPE carriers may cause difficulties for patient flow, as well as high costs due to the need for contact isolation. In order to prevent colonization, the investigation of the clinical predictors and risk factors for rectal CPE colonization admits of no delay. A recently published case-control study demonstrated solid organ and stem cell transplantation, mechanical ventilation, fecal incontinence, and exposure in the prior 30 days to carbapenems, vancomycin, and metronidazole as independent factors associated with CRE colonization [39]. In this prospective case-control study, operation of bronchoscopy, urinary system diseases, and combined use of antibiotics were demonstrated to be independently associated with rectal CPE colonization in highrisk patients from the ICU and HSCT wards. One possible explanation for bronchoscopy operation as an independent risk factor may be due to the fact that invasive bronchoscopy operation might have damaged the patients' natural immune barriers, and possibly led to CPE colonization. Given that invasive procedures such as the use of urinary catheters were frequently performed in patients with urinary system diseases, it is also easy to understand urinary system diseases as another independent risk factor for rectal CPE colonization. As invasive procedures were potential risk factors, infection control measures preventing the microbial colonization of the insertion sites are necessary. In addition, our study identified combined use of antibiotics as being associated with rectal CPE colonization. One possibility is that inappropriate combined antibiotic use may disrupt the gastrointestinal microflora and eradicate susceptible competing strains, thus elevating the incidence of $\mathrm{CPE}$ colonization [40].

Our study had several limitations. Firstly, this was a prospective single-center case-control study with relatively small sample size, and our results might not be applicable to other settings. Secondly, we didn't investigate the subsequent $\mathrm{CPE}$ infection rates in these CPEcolonized patients.

\section{Conclusion}

In conclusion, this study provided a detailed report of the prevalence, molecular epidemiology, and risk factors for rectal CPE colonization in high-risk patients from ICUs and HSCT wards in one medical center. Our data showed a high prevalence of rectal CPE colonization in high-risk patients from ICU and HSCT wards, and a predominant colonization of the ST11-type, KPC- 
producing K. pneumoniae strains. A bundle of infection control and prevention measures with an anti-infective stewardship program is urgently needed to reduce the rectal CPE colonization, especially in patients who were afflicted by urinary system diseases, have underwent bronchoscopy, and were previously exposed to combined antibiotic use.

\section{Abbreviations}

CLSI: Clinical and Laboratory Standards Institute; CRE: Carbapenem-resistant Enterobacteriaceae; CPE: Carbapenemase-producing Enterobacteriaceae; MIC: Minimum inhibitory concentration; WHO: World health organization; ETP: Ertapenem; IMP: Imipenem; MEM: Meropenem

\section{Acknowledgements}

We would like to thank all the enrolled patients for their participation in our study.

\section{Authors' contributions}

All the authors contributed to data analysis, drafting and revising the article, gave final approval of the version to be published, and agreed to be accountable for all aspects of the work. LY and SFH designed this study, participated in statistic calculations and interpretation of data, and drafted this manuscript. LY, JDS, and XYX collected the samples and performed the experiments.

\section{Funding}

This study was supported in part by the Science and Technology Research Program of Chongqing Municipal Education Commission (No. KJ1500235), and the Medical Research Program of Chongqing Health and Family Planning Commission (No. 2018MSXM009).

\section{Availability of data and materials}

All the data of this article is available from the corresponding author if reasonably requested.

\section{Ethics approval and consent to participate}

The data and samples analyzed in the present study were obtained in accordance with the standards and approved by the Chongqing Medical University Institutional Review Board and the Biomedical Ethics Committee. Informed consent was acquired from each patient.

\section{Consent for publication}

Not applicable.

\section{Competing interests}

The authors declare that they have no competing interests.

Received: 7 April 2020 Accepted: 15 September 2020

Published online: 23 September 2020

\section{References}

1. Durante-Mangoni E, Andini R, Zampino R. Management of carbapenem resistant Enterobacteriaceae infections. Clin Microbiol Infect. 2019;25(8):94350.

2. Righi E, Peri AM, Harris PN, Wailan AM, Liborio M, Lane SW, et al. Global prevalence of carbapenem resistance in neutropenic patients and association with mortality and carbapenem use: systematic review and meta-analysis. J Antimicrob Chemother. 2017;72(3):668-77.

3. Potter RF, D'Souza AW, Dantas G. The rapid spread of carbapenem-resistant Enterobacteriaceae. Drug Resist Updat. 2016;29:30-46.

4. World Health Organization. Essential medicines and health products. prioritization of pathogens to guide discovery, research and development of new antibiotics for drug-resistant bacterial infections, including tuberculosis. Geneva, Switzerland: WHO; 2017.

5. WHO. WHO publishes list of bacteria for which new antibiotics are urgently needed. WHO Media Centre; 2017. p. 1-4. doi:/entity/mediacentre/news/ releases/2017/bacteria-antibiotics-needed/en/index.html.
6. Nordmann P, Dortet L, Poirel L. Carbapenem resistance in Enterobacteriaceae: here is the storm! Trends Mol Med. 2012;18(5):263-72.

7. Bush K, Bradford PA. Epidemiology of $\beta$-lactamase-producing pathogens. Clin Microbiol Rev. 2020;33(2):e00047-19.

8. Nordmann P, Poirel L. The diffificult-to-control spread of carbapenemase producers among Enterobacteriaceae worldwide. Clin Microbiol Infect. 2014; 20:821-30.

9. Roberts LW, Harris PNA, Forde BM, Ben Zakour NL, Catchpoole E, StantonCook M, et al. Integrating multiple genomic technologies to investigate an outbreak of carbapenemase-producing Enterobacter hormaechei. Nat Commun. 2020;11(1):466.

10. Lutgring JD, Limbago BM. The problem of carbapenemase-producingcarbapenem-resistant-Enterobacteriaceae detection. J Clin Microbiol. 2016; 54(3):529-34.

11. Biedenbach DJ, Kazmierczak K, Bouchillon SK, Sahm DF, Bradford PA. In vitro activity of aztreonam-avibactam against a global collection of gramnegative pathogens from 2012 and 2013. Antimicrob Agents Chemother. 2015;59(7):4239-48.

12. Falcone M, Paterson D. Spotlight on ceftazidime/avibactam: a new option for MDR gram-negative infections. J Antimicrob Chemother. 2016;71(10): 2713-22.

13. Lomovskaya O, Sun D, Rubio-Aparicio D, Nelson K, Tsivkovski R, Griffith DC, et al. Vaborbactam: spectrum of beta-lactamase inhibition and impact of resistance mechanisms on activity in Enterobacteriaceae. Antimicrob Agents Chemother. 2017;61(11):e01443-517.

14. Haidar G, Clancy CJ, Chen L, Samanta P, Shields RK, Kreiswirth BN, et al. Identifying spectra of activity and therapeutic niches for ceftazidimeavibactam and imipenem-relebactam against carbapenem-resistant Enterobacteriaceae. Antimicrob Agents Chemother. 2017;61(9):e00642-717.

15. Monogue ML, Thabit AK, Hamada Y, Nicolau DP. Antibacterial efficacy of eravacycline in vivo against gram-positive and gram-negative organisms. Antimicrob Agents Chemother. 2016;60(8):5001-5.

16. Denervaud-Tendon V, Poirel L, Connolly LE, Krause KM, Nordmann P. Plazomicin activity against polymyxin-resistant Enterobacteriaceae, including MCR-1-producing isolates. J Antimicrob Chemother. 2017;72(10):2787-91.

17. Hackel MA, Tsuji M, Yamano Y, Echols R, Karlowsky JA, Sahm DF. In vitro activity of the siderophore cephalosporin, cefiderocol, against carbapenemnonsusceptible and multidrug-resistant isolates of gram-negative bacilli collected worldwide in 2014 to 2016. Antimicrob Agents Chemother. 2018; 62(2):e01968-17.

18. Alm RA, Johnstone MR, Lahiri SD. Characterization of Escherichia coli NDM isolates with decreased susceptibility to aztreonam/avibactam: role of a novel insertion in PBP3. J Antimicrob Chemother. 2015;70:1420-8.

19. Nelson K, Hemarajata P, Sun D, Rubio-Aparicio D, Tsivkovski R, Yang S, et al. Resistance to ceftazidime-avibactam is due to transposition of KPC in a porin-deficient strain of Klebsiella pneumoniae with increased efflux activity. Antimicrob Agents Chemother. 2017;61:e00989-17.

20. Schneider A, Coope C, Michie S, Puleston R, Hopkins S, Oliver I. Implementing a toolkit for the prevention, management and control of carbapenemase-producing Enterobacteriaceae in English acute hospitals trusts: a qualitative evaluation. BMC Health Serv Res. 2019:19:689.

21. Jamal AJ, Garcia-Jeldes F, Baqi M, Borgia S, Johnstone J, Katz K, et al. Infection prevention and control practices related to carbapenemaseproducing Enterobacteriaceae (CPE) in acute-care hospitals in Ontario, Canada. Infect Control Hosp Epidemiol. 2019:40(9):1-7.

22. Fournier S, Monteil C, Lepainteur M, Richard C, Brun-Buisson C, Jarlier V. Long-term control of carbapenemase-producing Enterobacteriaceae at the scale of a large French multihospital institution: a nine-year experience, France, 2004 to 2012. Euro Surveill. 2014;19(19):20802.

23. Del Bianco F, Morotti M, Zannoli S, Dirani G, Fantini M, Pedna MF, et al. Comparison of four commercial screening assays for the detection of blaKPC, blaNDM, blaIMP, blaVIM, and blaOXA48 in rectal secretion collected by swabs. Microorganisms. 2019;7(12):704

24. Pisney LM, Barron MA, Kassner E, Havens D, Madinger NE. Carbapenem resistant Enterobacteriaceae rectal screening during an outbreak of New Delhi Metallo- $\beta$-lactamase-producing Klebsiella pneumoniae at an acute care hospital. Infect Control Hosp Epidemiol. 2014;35:434-6.

25. Lubbert C, Becker-Rux D, Rodloff AC, Laudi S, Busch T, Kalsers UXBM. Colonization of liver transplant recipients with KPC-producing Klebsiella pneumoniae is associated with high infection rates and excess mortality: a case-control analysis. Infection. 2014;42:309-16. 
26. Tischendorf J, de Avila RA, Nasia SN. Risk of infection following colonization with carbapenem-resistant Enterobactericeae: a systematic review. Am J Infect Control. 2016;44:539-43.

27. Ambretti S, Bassetti M, Clerici P, Petrosillo N, Tumietto F, Viale P, et al. Screening for carriage of carbapenem resistant Enterobacteriaceae in settings of high endemicity: a position paper from an Italian working group on CRE infections. Antimicrob Resist Infect Control. 2019;8:136.

28. Paño Pardo JR, Serrano Villar S, Ramos Ramos JC, Pintado V. Infections caused by carbapenemase-producing Enterobacteriaceae: risk factors, clinical features and prognosis. Enferm Infecc Microbiol Clin. 2014;32(Suppl 4):41-8.

29. Zhao ZC, Xu XH, Liu MB, Wu J, Lin J, Li B. Fecal carriage of carbapenemresistant Enterobacteriaceae in a Chinese university hospital. Am J Infect Control. 2014;42(5):e61-4.

30. Liu Q, Liu L, Li Y, Chen X, Yan Q, Liu WE. Fecal carriage and epidemiology of carbapenem-resistant Enterobacteriaceae among hospitalized patients in a university hospital. Infect Drug Resist. 2019;12:3935-42.

31. Shanmugakani RK, Akeda Y, Sugawara Y, Laolerd W, Chaihongsa N, Sirichot $\mathrm{S}$, et al. PCR-dipstick-oriented surveillance and characterization of mcr-1and carbapenemase-carrying Enterobacteriaceae in a Thai hospital. Front Microbiol. 2019;10:149.

32. Khan MA, Mohamed AM, Faiz A, Ahmad J. Enterobacterial infection in Saudi Arabia: first record of Klebsiella pneumoniae with triple carbapenemase genes resistance. J Infect Dev Ctries. 2019;13(4):334-41.

33. Vannice K, Benoliel E, Kauber K, Brostrom-Smith C, Montgomery P, Kay M, et al. Notes from the field: clinical Klebsiella pneumoniae isolate with three carbapenem resistance genes associated with urology procedures-King County, Washington, 2018. MMWR Morb Mortal Wkly Rep. 2019;68:667-8.

34. Yigit H, Queenan AM, Anderson GJ, Domenech-Sanchez A, Biddle JW, Steward CD, et al. Novel carbapenem-hydrolyzing beta-lactamase, KPC-1, from a carbapenem-resistant strain of Klebsiella pneumoniae. Antimicrob Agents Chemother. 2001;45:1151-61.

35. David S, Reuter S, Harris SR, Glasner C, Feltwell T, Argimon S, et al. Epidemic of carbapenem-resistant Klebsiella pneumoniae in Europe is driven by nosocomial spread. Nat Microbiol. 2019;4:1919-29.

36. Hall JM, Corea E, Sanjeewani HD, Inglis TJ. Molecular mechanisms of betalactam resistance in carbapenemase-producing Klebsiella pneumoniae from Sri Lanka. J Med Microbiol. 2014;63:1087-92.

37. Guh AY, Bulens SN, Mu Y, Jacob JT, Reno J, Scott J, et al. Epidemiology of carbapenem-resistant Enterobacteriaceae in 7 US communities, 2012-2013. JAMA. 2015;314:1479-87.

38. Wei DD, Wan LG, Deng Q, Liu Y. Emergence of KPC-producing Klebsiella pneumoniae hypervirulent clone of capsular serotype K1 that belongs to sequence type 11 in mainland China. Diagn Microbiol Infect Dis. 2016;85: 192-4.

39. Mills JP, Talati NJ, Alby K, Han JH. The epidemiology of carbapenemresistant Klebsiella pneumoniae colonization and infection among long-term acute care hospital residents. Infect Control Hosp Epidemiol. 2016;37:55-60.

40. Li S, Jia X, Li C, Zou H, Liu H, Guo Y, et al. Carbapenem-resistant and cephalosporin-susceptible Pseudomonas aeruginosa: a notable phenotype in patients with bacteremia. Infect Drug Resist. 2018;11:1225-35.

\section{Publisher's Note}

Springer Nature remains neutral with regard to jurisdictional claims in published maps and institutional affiliations.

Ready to submit your research? Choose BMC and benefit from:
- fast, convenient online submission
- thorough peer review by experienced researchers in your field
- rapid publication on acceptance
- support for research data, including large and complex data types
- gold Open Access which fosters wider collaboration and increased citations
- maximum visibility for your research: over 100M website views per year
At BMC, research is always in progress.
Learn more biomedcentral.com/submissions

